Randomized control trials

\title{
Impact of diet and nutraceutical supplementation on inflammation in elderly people. Results from the RISTOMED study, an open-label randomized control trial ${ }^{\text {मै }}$
}

\author{
R. Ostan ${ }^{a}$, M.C. Béné ${ }^{b}$, L. Spazzafumo ${ }^{\text {, }}$, A. Pinto ${ }^{\mathrm{d}}$, L.M. Donini ${ }^{\mathrm{d}}$, F. Pryen ${ }^{\mathrm{e}}$, Z. Charrouf ${ }^{\mathrm{f}}$, \\ L. Valentini ${ }^{g}$, H. Lochs ${ }^{\text {h }}$, I. Bourdel-Marchasson ${ }^{\mathrm{i}, \mathrm{j}, \mathrm{k}}$, C. Blanc-Bisson ${ }^{\mathrm{i}, \mathrm{j}, \mathrm{k}}$, F. Buccolini ${ }^{\mathrm{l}}$, \\ P. Brigidi ${ }^{\mathrm{m}}$, C. Franceschi ${ }^{\text {a, n, o }}{ }^{\text {, P.A. d'Alessio }}{ }^{\text {p, * }}$
}

a Department of Experimental, Diagnostic and Specialty Medicine, University of Bologna, Via San Giacomo 12, 40126 Bologna, Italy

${ }^{\mathrm{b}}$ Hematology Laboratory, University Hospital CHU, 9 Quai Moncousu, 44000 Nantes, France

c Biostatistical Center INRCA, Via S. Margherita 5, 60100 Ancona, Italy

d Experimental Medicine Department, Sapienza University of Rome, P.le Aldo Moro 5, 00185 Rome, Italy

e Institute for Organic Synthesis and Photoreactivity, National Research Council of Italy, CNR, (ISOF), Via P. Gobetti 101, 40129 Bologna, Italy

${ }^{\mathrm{f}}$ Department of Chemistry, University Mohammed V-Agdal, Rabat, Morocco

${ }^{g}$ Department Gastroenterology and Hepatology, Charité-Universitätsmedizin Berlin, CCM, Charitéplatz 1, 10117 Berlin, Germany

h Medical University of Innsbruck, Innrain 52, 6020 Innsbruck, Austria

${ }^{\mathrm{i}} \mathrm{CHU}$ Bordeaux, Clinical Gerontology, France

j CNRS, RMSB, UMR 5536, France

${ }^{\mathrm{k}}$ University Bordeaux, RMSB, UMR 5536, Bordeaux, France

${ }^{1}$ R\&D, VoxNet CEO, Via Giovanni Paisiello 32, 00198 Rome, Italy

${ }^{\mathrm{m}}$ Department of Pharmacy and Biotechnology, University of Bologna, Via Belmeloro 6, 40126 Bologna, Italy

n IRCCS, Institute of Neurological Sciences, Via Altura 3, 40139 Bologna, Italy

${ }^{\circ}$ National Research Council of Italy, CNR, Institute for Organic Synthesis and Photoreactivity (ISOF), Via P. Gobetti 101, 40129 Bologna, Italy

p Biopark Campus Cancer, 1 mail du Pr Georges Mathé, 94807 Villejuif, France

\section{A R T I C L E I N F O}

\section{Article history:}

Received 27 February 2015

Accepted 26 June 2015

\section{Keywords:}

Nutraceutics

Diet

Inflammation

Ageing

$d$-Limonene

Probiotics

\begin{abstract}
S U M M A R Y
Background \& aims: Eating habits may influence the life span and the quality of ageing process by modulating inflammation. The RISTOMED project was developed to provide a personalized and balanced diet, enriched with or without nutraceutical compounds, to decrease and prevent inflammageing, oxidative stress and gut microbiota alteration in healthy elderly people. This paper focused on the effect on inflammation and metabolism markers after 56 days of RISTOMED diet alone or supplementation with three nutraceutical compounds.

Methods: A cohort of 125 healthy elderly subjects was recruited and randomized into 4 arms (Arm A, RISTOMED diet; Arm B, RISTOMED diet plus VSL\#3 probiotic blend; Arm C, RISTOMED diet plus AISA $d$ Limonene; Arm D, RISTOMED diet plus Argan oil). Inflammatory and metabolism parameters as well as the ratio between Clostridium cluster IV and Bifidobacteria (CL/B) were collected before and after 56 days of dietary intervention, and their evolution compared among the arms. Moreover, participants were subdivided according to their baseline inflammatory parameters (erythrocytes sedimentation rate (ESR), C-Reactive Protein, fibrinogen, Tumor Necrosis Factor-alfa (TNF- $\alpha$ ), and Interleukin 6) in two clusters with low or medium-high level of inflammation. The evolution of the measured parameters was then examined separately in each cluster.

Results: Overall, RISTOMED diet alone or with each nutraceutical supplementation significantly decreased ESR. RISTOMED diet supplemented with d-Limonene resulted in a decrease in fibrinogen, glucose, insulin levels and HOMA-IR. The most beneficial effects were observed in subjects with a medium-high inflammatory status who received RISTOMED diet with AISA $d$-Limonene supplementation. Moreover, RISTOMED diet associated with VSL\#3 probiotic blend induced a decrease in the CL/B ratio.
\end{abstract}

\footnotetext{
* Registered under ClinicalTrials.gov Identifier no. NCT01069445-NCT01179789.

* Corresponding author.

E-mail address: endocell@wanadoo.fr (P.A. d'Alessio).
} 
Conclusions: Overall, this study emphasizes the beneficial anti-inflammageing effect of RISTOMED diet supplemented with nutraceuticals to control the inflammatory status of elderly individuals.

(C) 2015 Elsevier Ltd and European Society for Clinical Nutrition and Metabolism.

\section{Introduction}

One of the typical aspects of the ageing process is the development of a low grade inflammatory status called "inflammageing" which plays a critical role in the pathogenesis of the main agerelated chronic diseases as Parkinson's and Alzheimer's diseases, type 2 diabetes, atherosclerosis, sarcopenia and cancer [1-3].

This inflammatory status includes a profound modification within the cytokine network leading to a general increase in plasma levels and cell capability to produce pro-inflammatory cytokines with a subsequent increase of the main inflammatory markers [4].

Inflammageing is the result of a dynamic signaling produced by different organs and tissues. In particular, adipose tissue has been recognized as an active endocrine organ able to regulate physiological and pathological processes, such as immunity and inflammation, through the production of pro-inflammatory and antiinflammatory factors, including cytokines (Interleukin-6, IL-6 and Tumor Necrosis Factor-alpha, TNF- $\alpha$ ), adipokines (leptin, adiponectin and resistin), and chemokines. The aging process is physiologically associated with a lean body mass loss leading to an increase in fat mass percentage on body weight, specifically in the abdominal region [5]. In this context, anti-inflammatory strategies may be useful to prevent or delay ageing and the onset of agerelated diseases [6].

The excessive intake of energy and nutrients together with the kind of nutrients consumed may turn on inflammatory pathways, directly or indirectly, through different mechanisms such as the induction of obesity and endoplasmic reticulum stress. In particular, dietary fatty acids may affect inflammatory processes notably through modulation of the metabolism of eicosanoids and of the expression of inflammatory cytokines through a series of transcriptional events mediated by NF- $\kappa \mathrm{B}$. Moreover, sugars and carbohydrates stimulate pancreatic islets and insulin-sensitive tissues such as adipose tissue, liver and muscles [7-9]. Conversely, malnutrition and, in particular, a diet with insufficient protein intake, is associated with frailty, decline in functional status, impaired muscle function, decreased bone mass, immune dysfunction, reduced cognition and mortality [9-11].

For all these reasons, assessment of the adequate dietary requirement and administration of the correct nutritional intake could be a fundamental issue to maintain or restore health in advanced age.

The European project RISTOMED (www.ristomed.eu), a multicenter 'open-label' randomized study (CORDIS FP7), was designed to develop and provide a healthy, personalized and balanced dietary intervention, enriched or not with specific nutraceutical compounds, to decrease and prevent inflammageing, oxidative stress and gut microbiota alteration in healthy elderly people. RISTOMED diet was built to reach the recommended daily requirement of nutrients, vitamins and minerals complying with different cultural patterns. RISTOMED diet was designed with a particular attention towards those foods and nutrients known or suspected to be able to reduce inflammation and oxidative stress by promoting a healthy gut microbiota in elderly people [12].

The aim of this study was to investigate the effect of RISTOMED diet supplemented or not with nutraceuticals on inflammageing and other critical processes (oxidative stress and gut microbiota alteration) in elderly healthy people, taking into account that the health of gut microbiota is associated with the immune functionality [13]. The primary outcome was the decrease of hsCRP (highsensitivity C-reactive protein) levels in each study arm. HsCRP was selected as primary outcome because it is an internationally recognized marker of inflammation and cardiovascular risk [14,15]. Secondary outcomes included other markers of inflammatory and metabolic status, oxidative stress, gut microbiota composition as well as quality of life and physical performance.

In the present paper, we report some results of the RISTOMED study, focusing on the effect of RISTOMED diet alone or with three nutraceutical compounds, namely a probiotic blend, an orange peel extract containing $d$-Limonene and Argan oil, on inflammatory and metabolic markers in healthy elderly subjects.

\section{Materials and methods}

\subsection{Recruitment}

It was planned to recruit 144 elderly subjects (age 65-85 years old, equal number of males and females) in three European cities (Rome, Italy; Bordeaux, France; Berlin, Germany), between May 2009 and April 2010, according to strict inclusion and exclusion criteria. Body Mass Index (BMI) was to be in the range of $22-30 \mathrm{~kg} /$ $\mathrm{m}^{2}$, in the absence of known diseases (neurologic or psychiatric disorder, geriatric anorexia, active infection, constipation and gastric disease requiring medical therapy, chronic inflammatory bowel disease, diabetes mellitus, dyslipidemia and/or other metabolic diseases, neoplasms). The subjects should not receive any chronic anti-inflammatory therapy such as NSAIDs nor any other kind of therapy in the 20 days before the beginning of the study. Participants had to use no food supplements or functional foods such as probiotics, prebiotics, vitamins and minerals in the week before the beginning of the study. Moreover, the subjects had to be able to comply with management of nutraceutical products, to use a computer and to access the web, by themselves or with the help of a caregiver. The study protocol was approved by the ethics committee at each recruiting institution and the participants gave written informed consent at enrollment. The study was registered at ClinicalTrials.gov as NCT01069445-NCT01179789, and was conducted under the 7th Framework Program of the European Commission.

After obtaining informed consent, the subjects were randomized into 4 arms: Arm A received the RISTOMED diet alone, Arm B received the RISTOMED diet supplemented with the VSL\#3 probiotic blend, Arm $C$ received the RISTOMED diet supplemented with the AISA-5203-L fruit peel extracted monoterpene $d$-Limonene, Arm D received the RISTOMED diet supplemented with Argan Oil. Randomization was performed centrally and stratified by recruitment center and gender as described elsewhere [12]. The study was conducted in accordance with International Good Clinical Practice guidelines (ICH, May 1977), the Declaration of Helsinki, and national laws and regulations.

All subjects were evaluated before starting the study (D0) and after a period of $56 \pm 2$ days of diet with or without supplementation (D56). A fasting venous blood sample and stools were 


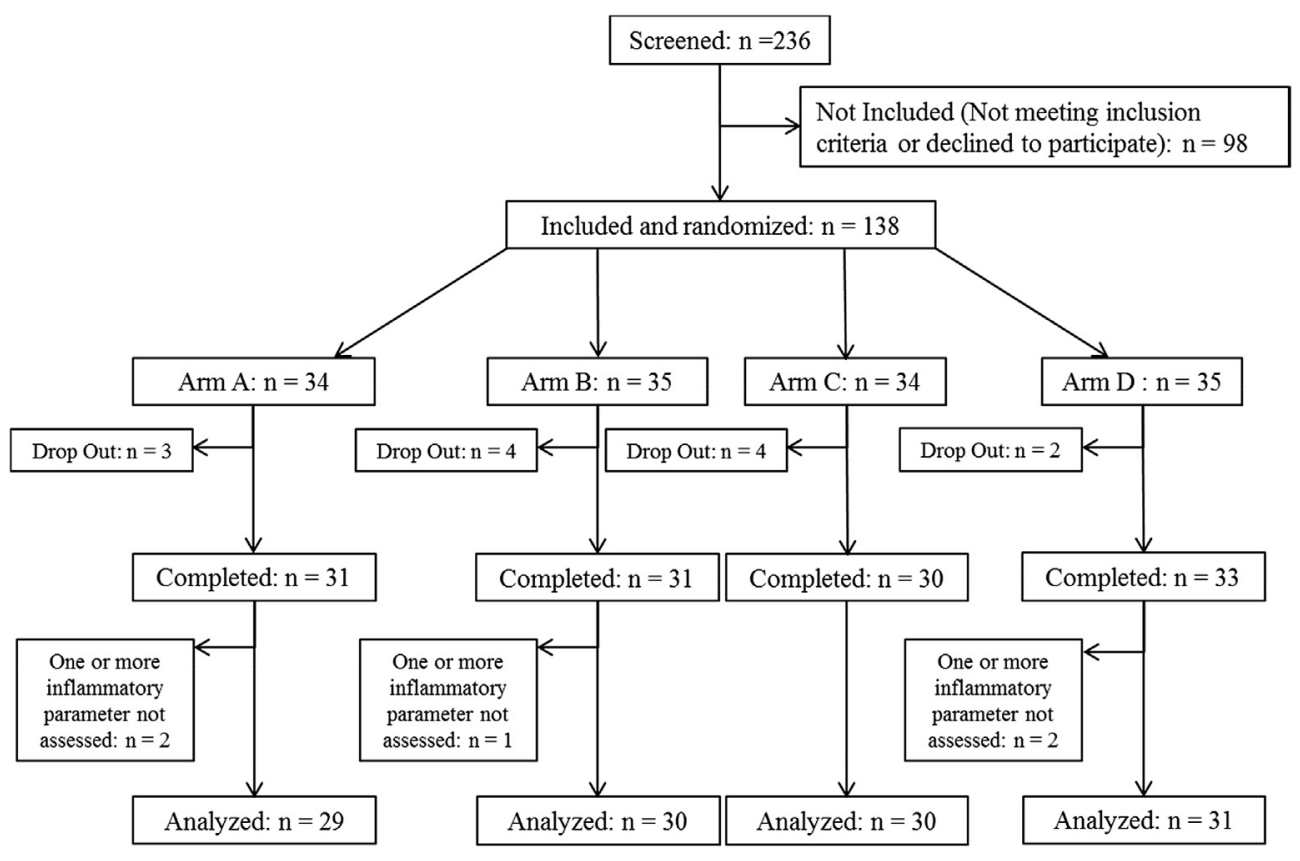

Fig. 1. Flow chart of participants of the RISTOMED project.

collected early in the morning before (D0) and after the diet period (D56).

\subsection{Diet}

The RISTOMED diet was personalized by a dietician using the RISTOMED-Health dietary Services (www.ristomed.eu) [12]. Each participant received a personal login to the RISTOMED website, and was trained by a dietician to get the personalized diet from the web platform. Recipes and daily menus were formulated taking into account traditional and personal eating habits. The nutritional pattern was established in agreement with the current dietary recommendations for elderly people, specific attention being given to the intake of food compounds that can affect inflammation, oxidative stress and gut microbiota [i.e. Poly Unsaturated Fatty Acids - PUFA n-6/n-3 ratio - antioxidant vitamins (vitamin E, C and carotenoids) and minerals (zinc and selenium), polyphenols and soluble dietary fiber].

The RISTOMED diet provided $\sim 15 \%$ of energy as proteins, $50-60 \%$ as carbohydrates and $\sim 35 \%$ as lipids. The latter were 15-20\% Mono-Unsaturated Fatty Acids (MUFA), provided by virgin olive oil, $~ 8 \%$ Saturated Fatty Acids (SFA) and $~ 7 \%$ PUFA with $0.5 \% \alpha$ linolenic acid and $2 \%$ linoleic acid. The daily recommended intake of uncooked Argan oil ( $25 \mathrm{~mL})$, when required in the study arm, was given in partial replacement of an equal amount of extra-virgin olive oil. Dietary fiber intake was $\sim 35 \mathrm{~g} /$ day with an insoluble/soluble fiber ratio of around $1: 1$, related to the prebiotic activity generally assigned to soluble fiber [16]. Polyphenols' intake was established at $\geq 400 \mathrm{mg} /$ day (as phenolic acids plus flavonoids;
[17]). The intake of vitamins and minerals was referenced to the WHO program "Keep fit for life" [18]. The minimum water need for elderly people is $1-1.5 \mathrm{~mL} / \mathrm{kcal} /$ day and it was recommended to use a calcium-rich water ( $\mathrm{Ca} \geq 150 \mathrm{mg} / \mathrm{L}$ ).

On the website, participants recorded their weight weekly and described the compliance to the diet and to the nutraceutical supplementation daily. After 2 and 6 weeks (D14 and D42) from the beginning of the intervention, the nutritionist/dietician contacted by phone each participant to verify his/her compliance to the diet and to the nutraceutical supplementation.

\subsection{Nutraceutical supplementation}

VSL\#3 probiotic blend (ACTIAL Farmaceutica Lda) is formulated as a granulated powder of 112 billion lyophilized bacteria per capsule in defined ratios of 4 strains of Lactobacillus (Lactobacillus paracasei, Lactobacillus plantarum, Lactobacillus acidophilus, and Lactobacillus delbrueckii subsp. bulgaricus), 3 strains of Bifidobacterium (Bifidobacterium longum, Bifidobacterium breve, and Bifidobacterium infantis) and Streptococcus thermophilus. The product was administered orally, 2 capsules daily on an empty stomach with a glass of water.

The monoterpene AISA 5203-L ( $d$-Limonene and its metabolites) extracted from orange peel was administrated orally in soft gel capsules with a meal and a large glass of water. The daily number of soft gel capsules was established for each patient according to the recommended dose of $10 \pm 1 \mathrm{mg} / \mathrm{kg}$.

Argan oil, the extra-virgin oil obtained using a cold-pressed technique of kernels contained in the Argania spinosa fruit, was

Table 1

Drop out for each arm of dietary intervention.

\begin{tabular}{lll}
\hline & No compliance & Adverse events \\
\hline Arm A & $n=1$ & $n=2$ (Dental infection - Chest tightness) \\
Arm B & $n=1$ & $n=3$ (Sleep disturbance - Stomach pain - Inflammatory status) \\
Arm C & $n=0$ & $n=4$ (Antibiotic therapy - Sigmoiditis - Nose bleeding - Nausea) \\
Arm D & $n=1$ & $n=1$ (Bowel discomfort) \\
\hline
\end{tabular}


Table 2



\begin{tabular}{|c|c|c|c|c|c|}
\hline & Arm A (diet) & Arm B (Diet + VSL\#3) & Arm C (Diet + AISA) & Arm D (Diet + Argan oil $)$ & $p$ \\
\hline $\mathrm{N}$ & 31 & 31 & 30 & 33 & \\
\hline Age, mean (SD) & $70.5(4)$ & $69.7(3.9)$ & $70(3.8)$ & $71.4(3.9)$ & NS \\
\hline Gender, $\mathrm{M} / \mathrm{F}$ & $14 / 17$ & $15 / 16$ & $14 / 16$ & $15 / 18$ & NS \\
\hline BMI, $\mathrm{kg} / \mathrm{m}^{2}(\mathrm{SD})$ & $26.9(3.4)$ & $26.7(3.8)$ & $26.8(2.8)$ & $26.6(3.5)$ & NS \\
\hline
\end{tabular}

supplied in $12.5 \mathrm{~mL}$ monodose sachets. The daily recommended intake was $25 \mathrm{~mL}$ taken as two monodoses per day at each person's convenience with breakfast, lunch and/or dinner.

\subsection{Laboratory analyses}

Peripheral blood analyses were performed on whole blood, serum or plasma collected as described above [12]. Standard clinical laboratory methods or specific ELISA tests were used (Aushon Biosystems, Billerica, MA; BioVendor, Modrice, Czech Republic; Mediagnost, Reutlingen, Germany). Inflammatory status was established using Erythrocytes Sedimentation Rate (ESR), hsCRP, fibrinogen, TNF- $\alpha$ and IL-6. Since changes in the gut microbiota composition may be connected with inflammageing, stool samples were collected on D0 and D56 to measure the ratio between Clostridium cluster IV and Bifidobacteria (CL/B) through 16S rRNA genetargeted qPCR [12].

Insulin resistance was assessed by measuring glucose and insulin levels and calculating the HOMA-IR index according to a previously published formula [19].

\subsection{Statistical analyses}

The effects of the dietary interventions measured on D56 were assessed as percentage variations compared to baseline, in order to minimize individual disparities. After testing for normality, these percentages of variation were compared between the four arms with a Kruskal-Wallis test. Paired Wilcoxon rank sum tests were used to assess the median percentages of variations for each parameter between D0 and D56 according to the arm of dietary intervention.

Two-Step Cluster Analysis was applied to determine 2 groups of subjects identified as "low inflammation" and "medium-high

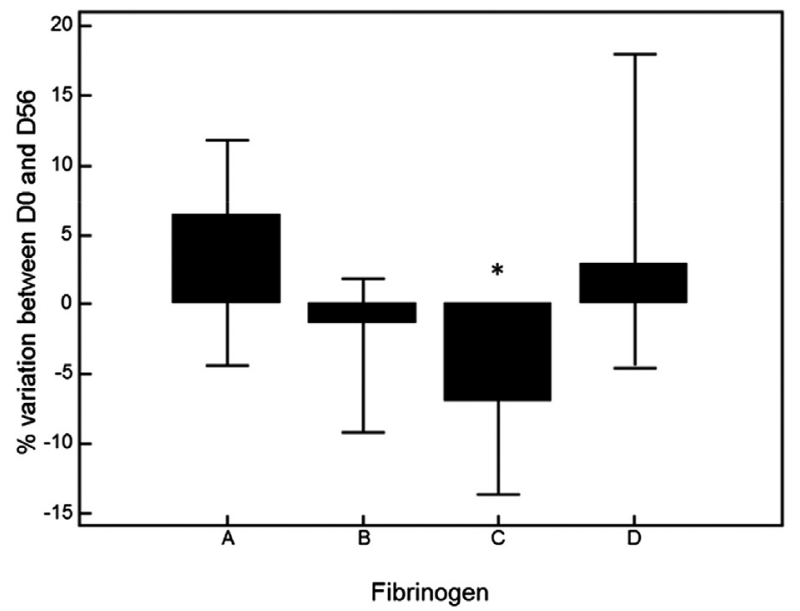

Fig. 2. Percentages of variation of fibrinogen levels among the arms of dietary intervention. $\mathrm{A}=$ RISTOMED diet, $\mathrm{B}=$ RISTOMED diet + VSL\#3, $\mathrm{C}=$ RISTOMED diet + AISA $\mathrm{D}=$ RISTOMED diet + Argan oil. Statistical analyses were performed by Kruskal-Wallis test; ${ }^{*} p<0.05$ between arm $C$ and arms A and D. inflammation" clusters using the inflammatory parameters described above at baseline. As Two-Step Cluster Analysis assumes a normal distribution of interval data the parameters were ztransformed to approximate normality. Baseline comparisons between the two inflammation clusters were performed using a Mann Whitney test. Kruskal-Wallis and paired Wilcoxon tests were applied as above in each cluster.

All tests were performed using the Medcalc ${ }^{\circledR}$ (bvba, Ostend, Belgium) software and SPSS/Win program version 19 (Spss Inc., Chicago, IL). The level of statistical significance was defined by a two-tailed $p$ value $<0.05$.

\section{Results}

\subsection{Global analysis}

A total of 125 elderly subjects (mean age $70.4 \pm 3.9 \mathrm{yrs}, 58$ males and 67 females) were recruited and completed the study. The flow chart of participants is reported in Fig. 1, drop outs for each arm are reported in Table 1 . No significant differences in age $(p=0.550)$ and gender distribution ( $p=0.568)$ were found between the three recruitment centers (data not shown). As shown in Table 2, the four arms of dietary intervention were comparable in age, gender distribution and BMI at baseline (D0). Compliance with the intake of nutraceuticals was between $87 \%$ and $95 \%$, between $71 \%$ and $80 \%$ and between $81 \%$ and $90 \%$ for VSL\#3, AISA, and Argan Oil respectively in different centers.

The differences between D0 and D56 were compared among the four arms of dietary intervention.

Although this was the primary outcome expected, hsCRP variations were not significant either between arms or within each of them (Arm A, median variation $-7.7 \%$, Arm B, 0\%, Arm C, $-7.1 \%$ and Arm D, 0\%, Kruskal-Wallis test $p=0.86)$.

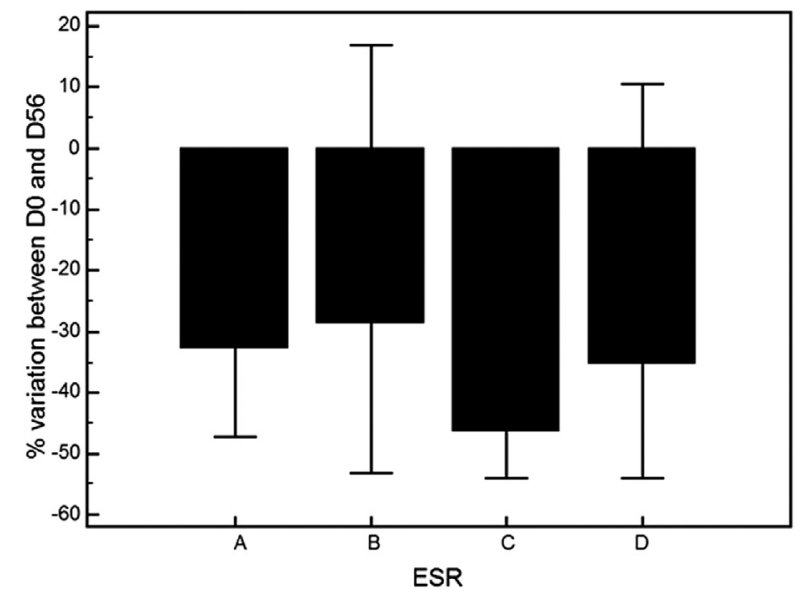

Fig. 3. Significant percentages of variation of ESR in each arm of dietary intervention. $\mathrm{A}=$ RISTOMED diet, $\mathrm{B}=$ RISTOMED diet + VSL\#3, $\mathrm{C}=$ RISTOMED diet + AISA $\mathrm{D}=$ RISTOMED diet + Argan oil. Statistical analyses were performed by paired Wilcoxon test between D0 and D56. $p$ values are reported in the text. 
Table 3

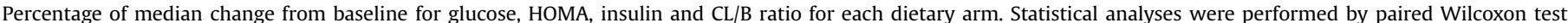
between D0 and D56.

\begin{tabular}{|c|c|c|c|c|c|c|c|c|}
\hline Change from baseline & $\operatorname{Arm~A}$ & $p$ & Arm B & $p$ & $\operatorname{Arm~C}$ & $p$ & Arm D & $p$ \\
\hline Glucose (mg/dL) & $-2.13 \%$ & 0.03 & $0 \%$ & 0.70 & $-4.35 \%$ & $<0.01$ & $+0.98 \%$ & 0.15 \\
\hline HOMA-IR & $-17.07 \%$ & 0.14 & $-7.94 \%$ & 0.26 & $-22.13 \%$ & 0.02 & $-2.87 \%$ & 0.50 \\
\hline Insulin $(\mu \mathrm{U} / \mathrm{mL})$ & $-10.00 \%$ & 0.26 & $-2.81 \%$ & 0.10 & $-20.40 \%$ & 0.04 & $-1.72 \%$ & 0.60 \\
\hline $\mathrm{CL} / \mathrm{B}$ ratio & $+26.40 \%$ & 0.48 & $-44.05 \%$ & 0.70 & $+14.30 \%$ & 0.80 & $+78.80 \%$ & 0.03 \\
\hline
\end{tabular}

Table 4

Clustering of the subjects according to their baseline inflammation status and dietary arm.

\begin{tabular}{|c|c|c|c|c|c|c|}
\hline Cluster & $n$ & Age, mean (S.D.) & $\operatorname{Arm} \mathrm{A}(n)$ & $\operatorname{Arm} B(n)$ & $\operatorname{Arm} C(n)$ & $\operatorname{Arm~D}(n)$ \\
\hline Low inflammation & 51 & $69.5(3.8)$ & 13 & 10 & 14 & 14 \\
\hline Medium inflammation & 69 & $70.9(3.9)$ & 16 & 20 & 16 & 17 \\
\hline
\end{tabular}

Fibrinogen concentration decreased significantly in Arm C (RISTOMED diet + AISA) compared to Arm A (RISTOMED diet) and Arm D (RISTOMED diet + Argan oil) ( $p=0.04$, Fig. 2).

The differences between D0 and D56 were then analyzed in each arm of dietary intervention.

ESR decreased significantly in each $\operatorname{arm}(\operatorname{Arm} A: p=0.02$, Arm B: $p=0.046$, Arm C: $p=0.02$, Arm D: $p=0.01$; Fig. 3).

Glucose decreased significantly in $\operatorname{Arm~A}(-2.13 \%, p=0.03)$ and in $\operatorname{Arm~C}(-4.35 \%, p=0.005)$. Insulin $(-20.4 \%, p=0.04)$ and HOMAIR $(-22.13 \%, p=0.02)$ also significantly decreased in Arm C and the CL/B ratio significantly increased by $79 \%(p=0.03)$ in Arm D (Table 3).

\subsection{Analysis according to the inflammation status at baseline}

As mentioned above, two-step cluster analysis based on available inflammatory parameters (ESR, hsCRP, fibrinogen, TNF- $\alpha$ and IL-6) at baseline (D0) identified two clusters of older subjects with low or medium-high level of inflammation (Table 4). The "low inflammation" cluster consisted of 51 subjects (mean age $69.5 \pm 3.76$ yrs, 23 males and 28 females). The "medium-high inflammation" cluster consisted of 69 subjects (mean age $70.9 \pm 3.93 \mathrm{yrs}, 27$ males and 42 females). Inflammatory parameters (except TNF- $\alpha$ ) were significantly higher in subjects belonging to the medium-high inflammation cluster compared to subjects in the low inflammation cluster (Table 5).

The effects on dietary interventions were then analyzed according to the inflammation status of the subjects in each arm of dietary intervention.

In the low inflammation cluster, minor response to the dietary interventions was seen. The only significant change, comparing the four arms by Kruskal-Wallis analysis, was increased fibrinogen levels in Arm D ( $+29.9 \%$ compared to all other groups, $p=0.03$ ). The percentage of variation between D0 and D56 was indeed highly significant in this group (paired Wilcoxon test, $p=0.007$ ). No significant changes in fibrinogen levels were seen in the other arms. Within arms analyses by paired Wilcoxon tests further showed that ESR significantly decreased in $\operatorname{Arm~A~}(-37 ; 6 \%, p=0.03)$, and that glucose decreased in $\operatorname{Arm} C(-6.3 \%, p=0.005$, data not shown).
In the medium-high inflammation cluster, the percentages of variation between D0 and D56, tested between arms by a Kruskal-Wallis test, showed that the CL/B ratio $(p=0.03)$ and IL-6 levels ( $p=0.04$ ) were significantly different (Fig. 4). Indeed, the $\mathrm{CL} / \mathrm{B}$ ratio was significantly decreased in Arm B (-46.2\%) compared to Arms C $(+95.5 \%)$ and D (+83.2\%) and IL-6 levels were significantly decreased in Arm C (-26.3\%) compared to Arms A (+17.3\%) and $\mathrm{B}(+15.9 \%)$.

Within arms analyses by paired Wilcoxon tests further showed that ESR decreased significantly in $\operatorname{Arm} B(-27 \%, p=0.049)$ and in Arm D ( $-49 \%, p=0.009)$ while the $C L / B$ ratio increased significantly in Arm D $(+83 \%, p=0.008)$ and $C(+95.5 \%, p=0.04$, data not shown). By contrast, inflammatory markers (fibrinogen $p=0.015$; IL-6, $p=0.009$ ) and insulin resistance parameters (insulin, $p=0.05$; HOMA-IR, $p=0.048$ ) significantly decreased in Arm C (Fig. 5).

\section{Discussion}

Observational studies have examined the association of the Mediterranean diet with inflammatory markers in healthy adults, reporting that this dietary regimen is able to reduce the expression of pro-inflammatory and proatherogenic genes in elderly people [20-22].

The innovative aspect of the European study RISTOMED was to design diets combining traditional foods and recipes with nutraceutical compounds allowing to assess their effectiveness in the context of a personalized balanced Mediterranean diet, the RISTOMED diet.

Here we show that RISTOMED diet and nutraceutical supplementation can significantly modulate inflammation and insulin resistance parameters, especially in healthy older people with a medium/high inflammatory status. Indeed, there is evidence in the literature that glucose metabolism and the inflammatory status are closely related [23]. Of note, among inflammation related parameters, we also considered gut microbiota modifications induced by dietary interventions and supplementation tested.

The anti-inflammatory effect of the RISTOMED diet, with or without supplementation, translated in a significant decrease of ESR in all arms. This parameter is a nonspecific indicator of infection and tissue injury often used to monitor chronic inflammation

Table 5

Definition of the "low" and "medium-high" inflammation clusters. Data are expressed as median (range). Statistical analyses were performed by Mann-Whitney test.

\begin{tabular}{|c|c|c|c|c|c|}
\hline Cluster & $\operatorname{ESR}(\mathrm{mm} / \mathrm{h})$ & $\mathrm{HsCRP}(\mathrm{mg} / \mathrm{L})$ & Fibrinogen $(\mathrm{mg} / \mathrm{dL})$ & $\mathrm{TNF}-\alpha(\mathrm{pg} / \mathrm{mL})$ & IL-6 (pg/mL) \\
\hline Low Inflammation & $8.1(2-25)$ & $1.2(0.2-9.3)$ & $295(180-420)$ & $0.7(0-18)$ & $7.3(0.01-18)$ \\
\hline Medium-high Inflammation & $30(6-90)$ & $2.7(0.3-25.2)$ & $419(299-710)$ & $1(0-723)$ & $14.7(2.7-367)$ \\
\hline$p$ & $<0.0001$ & $<0.0001$ & $<0.0001$ & 0.1 & $<0.0001$ \\
\hline
\end{tabular}



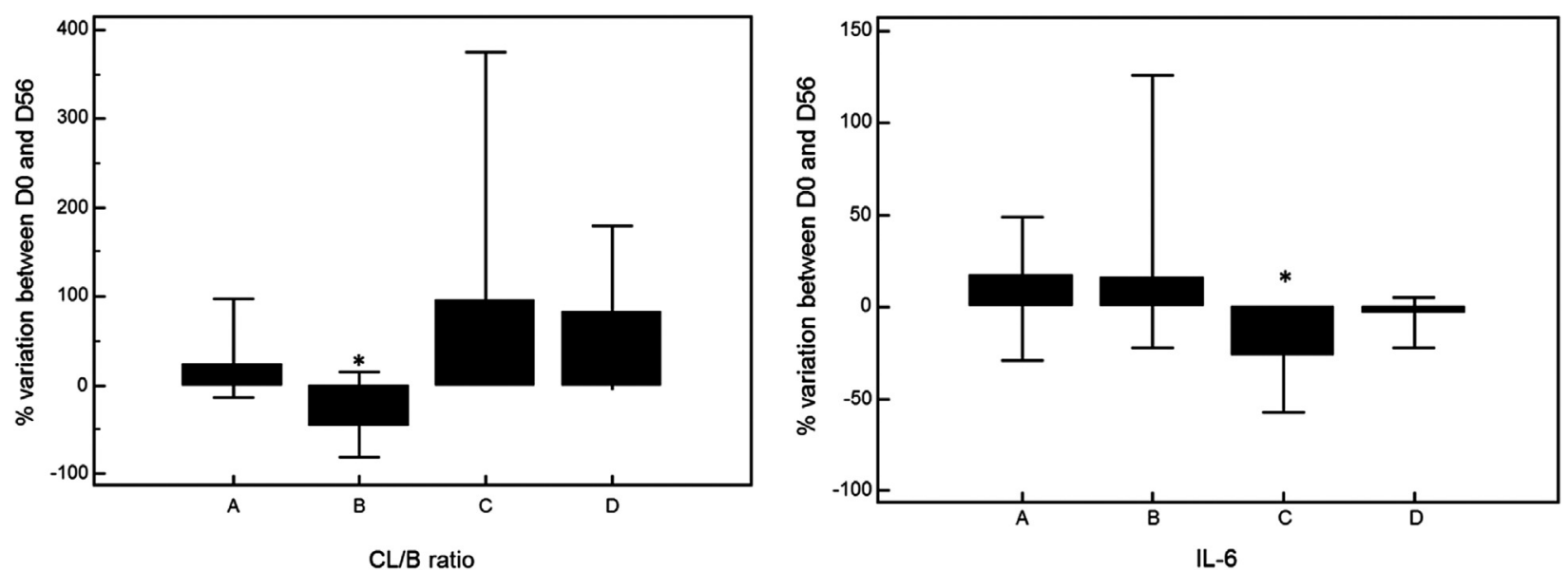

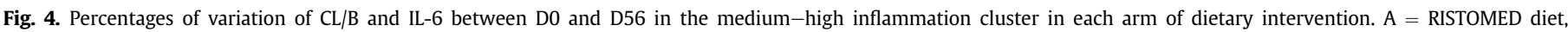
$\mathrm{B}=$ RISTOMED diet + VSL\#3, C = RISTOMED diet + AISA, D = RISTOMED diet + Argan oil. Statistical analyses were performed by Kruskal-Wallis test; ${ }^{*} p<0.05$.

and response to treatment in inflammatory diseases [24]. However, we did not find changes in hsCRP levels after the dietary interventions. A recent paper showed that CRP and the proteins that contribute to the ESR, such as fibrinogen, alpha-2 macroglobulin and immunoglobulins have distinct time courses and kinetics. Thus, CRP can rise and then fall at a different rate than ESR explaining the disagreements between these measures [25].

RISTOMED diet supplemented with natural $d$-Limonene extracted from orange peel decreased significantly fibrinogen levels. Of interest, this dietary and nutraceutical intervention affected significantly the largest number of parameters, with a major impact on insulin resistance markers.

When the subjects were further subdivided according to their baseline inflammatory profile, it is notable that only very few parameters were modified by the RISTOMED diet, stressing the inability to show an improvement in healthy not inflamed old individuals, using the inflammatory biomarkers selected in the RISTOMED study. Conversely, for subjects presenting a clear "inflammageing" profile, RISTOMED diet supplemented with $d$ Limonene significantly impacted on both inflammation and insulin resistance markers.

This study interestingly included investigation of variations of the microbiome concentrating on Clostridium cluster IV and



Fig. 5. Significant variations of inflammatory (fibrinogen and IL-6) and insulin resistance parameters (insulin and HOMA-IR) in the medium-high inflammation cluster in Arm C (RISTOMED diet + AISA) between D0 and D56. Statistical analyses were performed by paired Wilcoxon test between D0 and D56. $p$ values are reported in the text.
Bifidobacteria spp. In a previous paper, it was shown that RISTOMED diet supplemented with VSL\#3 probiotic blend increased Bifidobacteria and supported adequate folate and vitamin B12 concentrations decreasing homocysteine levels and suggesting a protective role for aging associated conditions, e.g. cardiovascular or neurodegenerative diseases [12]. Here we confirmed that the RISTOMED diet associated with VSL\#3 probiotic blend induced a decrease of the ratio between Clostridium cluster IV and Bifidobacteria, suggesting a better survival of Bifidobacteria spp. Conversely, the ratio showed a trend for increase in the other arms. When subdividing the subjects according to their baseline inflammatory status, striking differences were noted. RISTOMED diet supplemented with VSL\#3 probiotic blend tended to increase the ratio between Clostridium cluster IV and Bifidobacteria in the "low inflammation group" and to lower it in the "medium-high inflammation" group, while RISTOMED diet supplemented with $d$ Limonene had the reverse effect. These variations could be explained by the different targets of probiotics, acting directly on the intestinal microbiome, and $d$-Limonene, modifying the intestinal barrier function as reported elsewhere [26].

In conclusion, in spite of the relatively small size of the subgroups and dispersion of data, non-parametric analysis allowed to demonstrate that a balanced Mediterranean dietary regimen can counteract inflammageing, especially in elderly individuals with already altered inflammation parameters, on a prevention perspective. A screening in search of such latent inflammation might guide towards the most appropriate personalized approach. It moreover suggests that $d$-Limonene supplementation in the context of this dietary intervention could have a beneficial effect on older people in limiting the negative influence of chronic inflammation as demonstrated in other models [27] and improving insulin resistance parameters.

\section{Conflict of interest}

None

\section{Acknowledgments}

The RISTOMED study was funded by the 7th Framework Program of the European Union FP7-SME-2007-1 grant agreement 222230. 


\section{Appendix A. Supplementary material}

Supplementary data related to this article can be found at http:// dx.doi.org/10.1016/j.clnu.2015.06.010.

\section{References}

[1] Franceschi C, Bonafè M, Valensin S, Olivieri F, De Luca M, Ottaviani E, et al. Inflamm-aging. An evolutionary perspective on immunosenescence. Ann N Y Acad Sci 2000;908:244-54.

[2] Franceschi C, Capri M, Monti D, Giunta S, Olivieri F, Sevini F, et al. Inflammageing and anti-inflammageing: a systemic perspective on aging and longevity emerged from studies in humans. Mech Ageing Dev 2007;128: 92-105.

[3] De Martinis M, Franceschi C, Monti D, Ginaldi L. Inflammation markers predicting frailty and mortality in the elderly. Exp Mol Pathol 2006;80:219-27.

[4] Ostan R, Bucci L, Capri M, Salvioli S, Scurti M, Pini E, et al. Immunosenescence and immunogenetics of human longevity. Neuroimmunomodulation 2008;15: 224-40.

[5] Chang SH, Beason TS, Hunleth JM, Colditz GA. A systematic review of body fat distribution and mortality in older people. Maturitas 2012;72:175-91.

[6] Cevenini E, Caruso C, Candore G, Capri M, Nuzzo D, Duro G, et al. Age-related inflammation: the contribution of different organs, tissues and systems. How to face it for therapeutic approaches. Curr Pharm Des 2010;16:609-18.

[7] Hotamisligil GS. Inflammation and metabolic disorders. Nature 2006;444: $860-7$.

[8] de Heredia FP, Gómez-Martínez S, Marcos A. Obesity, inflammation and the immune system. Proc Nutr Soc 2012;71:332-8.

[9] Calder PC, Ahluwalia N, Brouns F, Buetler T, Clement K, Cunningham K, et al. Dietary factors and low-grade inflammation in relation to overweight and obesity. Br J Nutr 2011;106(Suppl 3):S5-78.

[10] Kelaiditi E, Guyonnet S, Cesari M. Is nutrition important to postpone frailty? Curr Opin Clin Nutr Metab Care 2015;18:37-42.

[11] Ahmed T, Haboubi N. Assessment and management of nutrition in older people and its importance to health. Clin Interv Aging 2010;5:207-16.

[12] Valentini L, Pinto A, Bourdel-Marchasson I, Ostan R, Brigidi P, Turroni S, et al. Impact of personalized diet and probiotic supplementation on inflammation, nutritional parameters and intestinal microbiota - the "RISTOMED project": randomized controlled trial in healthy older people. Clin Nutr 2014;34: 593-602.

[13] Guigoz Y, Doré J, Schiffrin EJ. The inflammatory status of old age can be nurtured from the intestinal environment. Curr Opin Clin Nutr Metab Care 2008;11:13-20.
[14] Pearson TA, Mensah GA, Alexander RW, Anderson JL, Cannon 3rd RO, Criqui M, et al. Centers for disease control and prevention; American Heart Association. Markers of inflammation and cardiovascular disease: application to clinical and public health practice. A statement for healthcare professionals from the centers for disease control and prevention and the American heart Association. Circulation 2003;107:499-511.

[15] Ballantyne CM, Nambi V. Markers of inflammation and their clinical significance. Atherosclerosis 2005; (Suppl 6):21-9.

[16] Ma Y, Griffith JA, Chasan-Taber L, Olendzki BC, Jackson E, Stanek 3rd EJ, et al. Association between dietary fiber and serum C-reactive protein. Am J Clin Nutr 2006;83:760-6.

[17] Mantovani G, Madeddu C, Maccio A, Gramignano G, Lusso MR, Massa E, et al. Cancer-related Anorexia/Cachexia syndrome and oxidative stress: an innovative approach beyond current treatment. Cancer Epidemiol Biomark Prev 2004; $13: 1651-95$.

[18] Keep fit for life - meeting the nutritional needs of older persons. World Health Organization - Tufts University School of Nutrition and Policy; 2002.

[19] Matthews DR, Hosker JP, Rudenski AS, Naylor BA, Treacher DF, Turner RC. Homeostasis model assessment: insulin resistance and beta-cell function from fasting plasma glucose and insulin concentrations in man. Diabetologia $1985 ; 28: 412-9$.

[20] Chrysohoou C, Panagiotakos DB, Pitsavos C, Das UN, Stefanadis C. Adherence to the Mediterranean diet attenuates inflammation and coagulation process in healthy adults: the ATTICA Study. J Am Coll Cardiol 2004;44:152-8.

[21] Azzini E, Polito A, Fumagalli A, Intorre F, Venneria E, Durazzo A, et al. Mediterranean diet Effect: an Italian picture. Nutr J 2011;10:125.

[22] Scoditti E, Calabriso N, Massaro M, Pellegrino M, Storelli C, Martines G, et al, Mediterranean diet polyphenols reduce inflammatory angiogenesis through MMP-9 and COX-2 inhibition in human vascular endothelial cells: a potentially protective mechanism in atherosclerotic vascular disease and cancer. Arch Biochem Biophys 2012;527:81-9.

[23] Collier B, Dossett LA, May AK, Diaz JJ. Glucose control and the inflammatory response. Nutr Clin Pract 2008;23:3-15.

[24] Weinstein A. The clinical and research significance of the erythrocyte sedimentation rate. J Rheumatol 1995;22:788.

[25] Sbong S, Feldman M. Frequency and causes of C-reactive protein and erythrocyte sedimentation rate disagreements in adults. Int J Rheum Dis 2015;18: 29-32.

[26] d'Alessio PA, Ostan R, Bisson JF, Schulzke JD, Ursini MV, Béné MC. Oral administration of D-Limonene controls inflammation in rat colitis and displays anti-inflammatory properties as diet supplementation in humans. Life Sci 2013;92:1151-6.

[27] d'Alessio PA, Mirshahi M, Bisson JF, Bene MC. Skin repair properties of DLimonene and perillyl alcohol in murine models. Antiinflamm Antiallergy Agents Med Chem 2014;13:29-35. 